\title{
A New Facile and Rapid Synthesis of Aliphatic Polyamides by Microwave-Assisted Polycondensation of $\omega$-Amino Acids and Nylon Salts
}

\author{
Yoshio ImaI, ${ }^{*}$ Hisashi Nemoto, Shinji Watanabe, and Masa-aki KaKimoto \\ Department of Organic and Polymeric Materials, Tokyo Institute of Technology, \\ Meguro-ku, Tokyo 152, Japan
}

(Received July 31, 1995)

\begin{abstract}
A new facile method for the rapid synthesis of aliphatic polyamides was developed by using a domestic microwave oven by the polycondensation of both $\omega$-amino acids and nylon salts in the presence of a small amount of a polar organic medium that acts as a primary microwave absorber. Suitable organic media were tetramethylene sulfone, amide-type solvents such as $N$-cyclohexyl-2-pyrrolidone and 1,3-dimethyl-2-imidazolidone, and phenolic solvents like $m$-cresol and $o$-chlorophenol. The polycondensation proceeded rapidly, compared with the conventional melt polycondensation, and was almost complete within $5 \mathrm{~min}$, producing a series of polyamides with inherent viscosities around $0.5 \mathrm{dl} \mathrm{g}^{-1}$.

KEY WORDS Microwave-Assisted Polycondensation / Aliphatic Polyamides / Rapid Polyamide Synthesis / Microwave Oven /
\end{abstract}

Recently there has been growing interest in applying microwave energy to synthetic organic chemistry and synthetic polymer chemistry as well. In the latter field, microwave energy has been utilized for the radical polymerization of vinyl monomers such as 2-hydroxyethyl methacrylate, ${ }^{1}$ methyl methacrylate, ${ }^{2}$ and styrene ${ }^{3}$ and for the curing of polymers such as epoxy resins ${ }^{4-10}$ and polyurethanes, ${ }^{1,12}$ as well as for the imidization of polyamic acids. ${ }^{13,14}$ Among them, much efforts have been directed toward the curing of epoxy resins from the practical viewpoint. In most cases, the high heat efficiency gave rise to remarkable rate enhancements and dramatic reduction of reaction times. However, there is no report so far except for our studies $^{15,16}$ on the synthesis of condensation polymers by using microwave energy. We have already reported the rapid synthesis of aromatic polyamides by microwave-assisted direct polycondensation of aromatic diamines and aromatic dicarboxylic acids with condensing agents, ${ }^{15}$ and a preliminary study on the microwave-assisted rapid synthesis of polyamides from nylon salts. ${ }^{16}$

This paper reports a facile method for the rapid synthesis of aliphatic polyamides by using a microwave oven by the polycondensation of both $\omega$-amino acids and nylon salts in more detail (Scheme 1). ${ }^{17,18}$

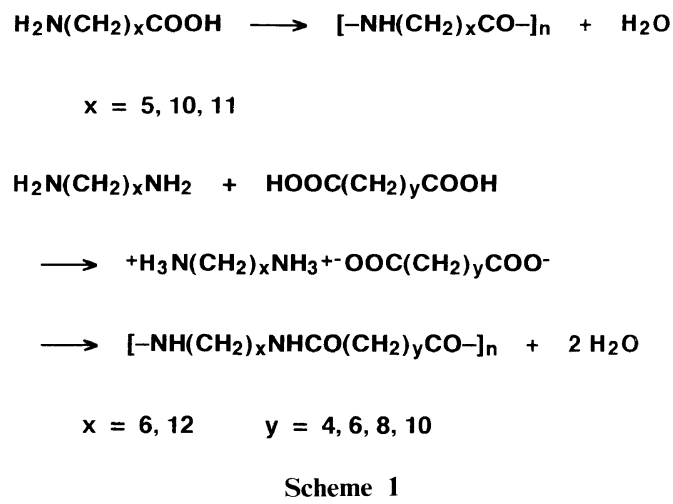

* To whom all correspondence should be addressed.

\section{EXPERIMENTAL}

\section{Apparatus}

The apparatus used for the polycondensation was a Mitsubishi RR-32 domestic microwave oven $(500 \mathrm{~W}$, $2.45 \mathrm{GHz}$ ). We made a minor modification to drill a small hole on the top of the microwave oven, and adopted an open reaction system using a $30 \mathrm{ml}$ wide-mouth vial as a reaction vessel.

\section{Monomers and Reagents}

Commercial 6-aminohexanoic acid ( $\varepsilon$-aminocaproic acid) and 11-aminoundecanoic acid were purified by recrystallization with water. Hexamethylenediamine and dodecamethylenediamine were obtained commercially and purified by distillation under reduced pressure. 12-Aminododecanoic acid, hexanedioic acid (adipic acid), octanedioic acid (suberic acid), decanedioic acid (sebacic acid), and dodecanedioic acid, as well as the solvents employed for the polymerization, were obtained commercially and used without further purification.

A series of nylon salts were prepared by a usual procedure $^{19}$ by mixing an ethanol solution of an aliphatic diamine with an ethanol solution of an aliphatic dicarboxylic acid, followed by recrystallization of the resultant precipitates with water. The melting point and elemental analysis data of the nylon salts prepared are tabulated in Table I.

\section{Polymerization Procedure}

The reaction vessel containing a mixture of a reactant and a solvent was placed on the center of the turn table in the microwave oven, then nitrogen gas was introduced from the top of the reaction vessel through a thin Teflon tube to minimize danger of fire in the reaction system. The mixture was irradiated with microwave for a prescribed time. The temperature of the reaction mixture was determined just immediately after removal of the reaction vessel from the microwave oven by using a thermocouple and a temperature recorder. The polymer formed was isolated by washing the reaction mixture with methanol, followed by drying under vacuum. The 
Table I. Melting point and elemental analysis data of nylon salts

\begin{tabular}{|c|c|c|c|c|c|c|c|}
\hline \multirow{3}{*}{$\begin{array}{l}\text { Nylon } \\
\text { salt }\end{array}$} & \multirow{3}{*}{$\mathrm{mp}^{\mathrm{a}} /{ }^{\circ} \mathrm{C}$} & \multicolumn{6}{|c|}{ Elememtal analysis $/ \%$} \\
\hline & & \multicolumn{3}{|c|}{ Calcd } & \multicolumn{3}{|c|}{ Found } \\
\hline & & $\mathrm{C}$ & $\mathrm{H}$ & $\mathrm{N}$ & $\mathrm{C}$ & $\mathrm{H}$ & $\mathrm{N}$ \\
\hline 66 & $204(192)$ & 54.94 & 9.99 & 10.68 & 54.63 & 10.26 & 10.52 \\
\hline 68 & 183 & 57.90 & 10.41 & 9.65 & 57.59 & 10.80 & 9.68 \\
\hline 610 & $179(171)$ & 60.35 & 10.76 & 8.80 & 59.88 & 10.93 & 8.72 \\
\hline 612 & 189 & 62.39 & 11.05 & 8.08 & 62.17 & 11.37 & 7.97 \\
\hline 126 & 156 & 62.39 & 11.05 & 8.08 & 61.97 & 11.42 & 8.01 \\
\hline 128 & 153 & 64.13 & 11.30 & 7.48 & 64.56 & 11.61 & 7.46 \\
\hline 1210 & $167(163)$ & 65.63 & 11.52 & 6.96 & 65.70 & 12.04 & 6.83 \\
\hline 1212 & 187 & 66.93 & 11.70 & 6.50 & 66.88 & 12.00 & 6.45 \\
\hline
\end{tabular}

${ }^{a}$ Determined by DTA at a heating rate of $10^{\circ} \mathrm{C} \mathrm{min}^{-1}$ in nitrogen. The mp given in parentheses is that reported in the literature. ${ }^{20}$

formation of polyamides was confirmed by means of.IR spectroscopy, and the IR spectra exhibited characteristic amide absorption bands at around $3440 \mathrm{~cm}^{-1}(\mathrm{~N}-\mathrm{H})$ and $1640 \mathrm{~cm}^{-1}(\mathrm{C}=\mathrm{O})$.

\section{Measurements}

IR spectra were recorded on a Shimadzu FT/IR-8100 spectrophotometer. Differential thermal analysis (DTA) was performed with a Shimadzu thermal analyzer DTA-40. Inherent viscosity of the polymer was measured by using a Ostwald viscometer at a concentration of $0.5 \mathrm{~g} \mathrm{dl}^{-1}$ in $m$-cresol at $30^{\circ} \mathrm{C}$.

\section{RESULTS AND DISCUSSION}

\section{Microwave Heating of Solvents}

It is well known that the heat generation by microwave irradiation is proportional to the product of dielectric constant $\varepsilon$ and dielectric loss tangent $\tan \delta$ of the material. ${ }^{4}$ It is also known that a material of a larger dielectric constant $\varepsilon$ generally has a larger dielectric loss tangent $\tan \delta$. Hence a polar material generates much heat quickly by microwave irradiation.

Figure 1 shows the time dependence of the temperature reached by the microwave irradiation on $2 \mathrm{ml}$ of various solvents. It is obvious that highly polar solvents such as water, 1,3-dimethyl-2-imidazolidone (DMI), and tetramethylene sulfone (sulfolane) generated heat quickly by the microwave irradiation. For example, water $(\varepsilon=78)$ was heated within $1 \mathrm{~min}$ to go up to the boiling point near $100^{\circ} \mathrm{C}$. Highly polar and high-boilingpoint solvents such as DMI $\left(\varepsilon=37\right.$ and bp $\left.225^{\circ} \mathrm{C}\right)$ and sulfolane $\left(\varepsilon=43\right.$ and bp $\left.287^{\circ} \mathrm{C}\right)$ were heated to $180^{\circ} \mathrm{C}$ after $1 \mathrm{~min}$ of the microwave irradiation. The heat generation of less polar $m$-cresol $\left(\varepsilon=12\right.$ and bp $\left.202^{\circ} \mathrm{C}\right)$ was intermediate between that of water and DMI or sulfolane.

\section{Polyamide Synthesis from $\omega$-Amino Acids}

Our strategy for the successful rapid synthesis of polyamides is the use of a small amount of a polar organic medium that acts as a primary microwave absorber. The organic medium, which again acts as a solvent for both the starting monomers and the resultant polyamides, allows to induce effective homogeneous heating of the monomers and thereby subsequent poly-

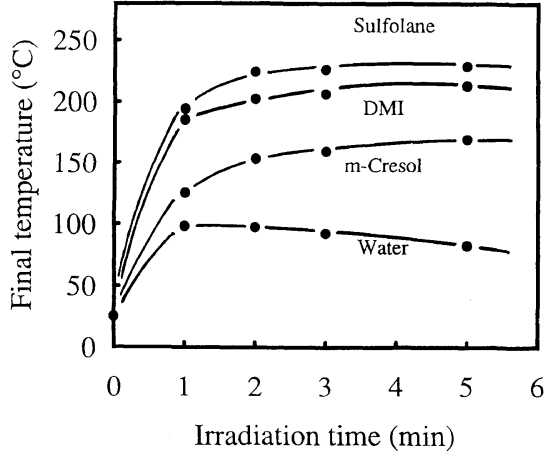

Figure 1. Time dependence of final temperature of various solvents $(2 \mathrm{ml})$ under microwave irradiation.

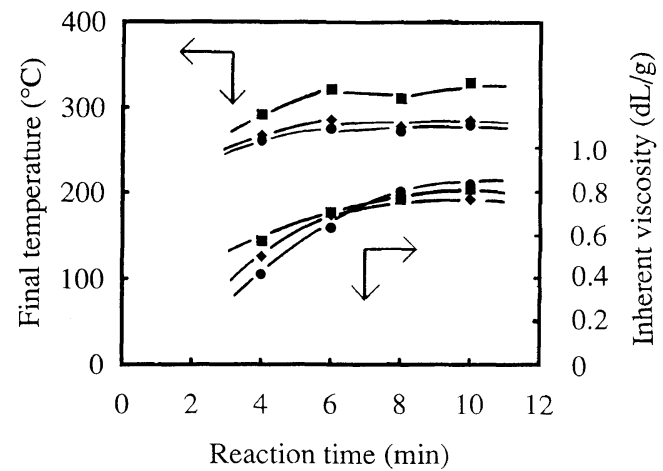

Figure 2. Time dependence of final temperature and inherent viscosity of the polyamide formed by the microwave-assisted polycondensation of 12-aminododecanoic acid $(2 \mathrm{~g})$ in different solvents $(2 \mathrm{ml})$ :

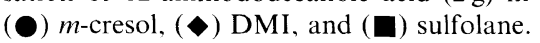

condensation leading to polyamides. Figure 2 exhibits the solvent effect on the polymerization temperature and the inherent viscosity of the polyamide formed by the polycondensation of 12-aminododecanoic acid as a function of microwave irradiation time. Here we used $m$-cresol, DMI, and sulfolane as solvents. The temperature of the reaction mixture rose quickly and reached above $270^{\circ} \mathrm{C}$ after $4 \mathrm{~min}$ of the microwave irradiation. At that time, most of the solvent evaporated from the polymerization mixture, leaving a melt (melting temperature of the polymer is $179^{\circ} \mathrm{C}$ ). During this initial period, the polycondensation proceeded rapidly, producing the polyamide having inherent viscosities of around $0.4 \mathrm{dl} \mathrm{g}^{-1}$. Between $4 \mathrm{~min}$ and $10 \mathrm{~min}$, the reaction temperature was maintained almost constant, while the inherent viscosity of the polymer gradually increased, finally reaching to about $0.7 \mathrm{dlg}^{-1}$.

Figure 3 shows the influence of the amount of $m$-cresol as the solvent, that is, the monomer concentration dependence, on the polymerization temperature and the inherent viscosity of the polyamide formed as a function of polymerization time. When a small amount of $0.5 \mathrm{ml}$ of $m$-cresol was used, the polymerization temperature reached to $250^{\circ} \mathrm{C}$ within $4 \mathrm{~min}$, giving readily the polyamide having an inherent viscosity above $0.6 \mathrm{dlg}^{-1}$. In the case of the polycondensation using a larger amount between 1.0 and $4.0 \mathrm{ml}$ of the solvent, the viscosity value of the polyamide increased from about 0.3 to $0.7 \mathrm{dl} \mathrm{g}^{-1}$ with increasing microwave irradiation time from 4 to $10 \mathrm{~min}$ after a short induction period for the polycondensation. 


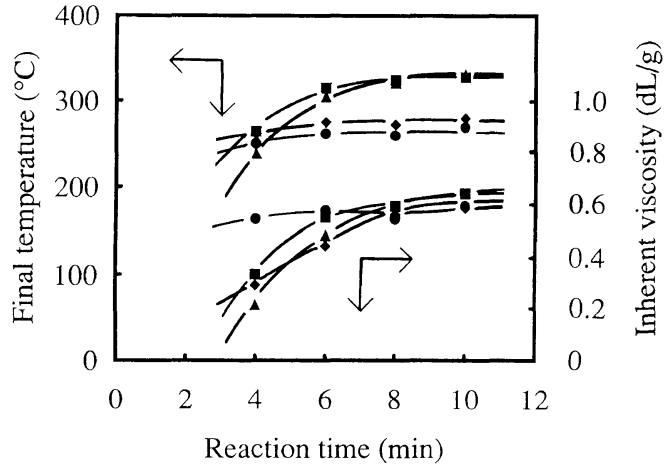

Figure 3. Time dependence of final temperature and inherent viscosity of the polyamide formed by the microwave-assisted polycondensation of 12-aminododecanoic acid (2g) in DMI solvent at different monomer concentration: $(\bullet) 0.5 \mathrm{ml},(\bullet) 1.0 \mathrm{ml},(\boldsymbol{\square}) 2.0 \mathrm{ml}$, and $(\boldsymbol{\Delta})$ $4.0 \mathrm{ml}$.

Table II. Solvent effect on microwave-assisted polycondensation of 12-aminododecanoic acid

\begin{tabular}{|c|c|c|c|c|c|c|}
\hline \multicolumn{4}{|l|}{ Solvent } & \multicolumn{2}{|c|}{ Reaction } & \multirow{2}{*}{$\begin{array}{c}\text { Polymer } \\
\eta_{\text {inh }}{ }^{\mathrm{d}}\end{array}$} \\
\hline \multirow{2}{*}{ Type } & \multirow{2}{*}{$\varepsilon$} & bp & $\mathrm{Ft}^{\mathrm{b}}$ & Time & $\mathrm{Ft}^{\mathrm{c}}$ & \\
\hline & & $\mathrm{C}$ & ${ }^{\circ} \mathrm{C}$ & $\min$ & ${ }^{\circ} \mathrm{C}$ & $\mathrm{dl} \mathrm{g}^{-1}$ \\
\hline Water & 78 & 100 & 97 & 5 & 220 & 0.35 \\
\hline Dimethyl sulfoxide & 47 & 189 & 172 & 4 & 259 & 0.24 \\
\hline Tetramethylene sulfone & 43 & 287 & 224 & 5 & 282 & 0.53 \\
\hline Dimethylacetamide & 38 & 166 & 163 & 5 & 281 & 0.23 \\
\hline 1,3-Dimethylimidazolidone & 37 & 225 & 202 & 5 & 300 & 0.60 \\
\hline$N$-Methylpyrrolidone & 32 & 202 & 179 & 4 & 267 & 0.39 \\
\hline$N$-Cyclohexylpyrrolidone & - & $\ldots$ & 224 & 5 & 266 & 0.46 \\
\hline Nitrobenzene & 35 & 211 & 198 & 5 & 264 & 0.42 \\
\hline Ethanediol & 38 & 197 & 193 & 5 & 317 & 0.59 \\
\hline 1,4-Butanediol & 31 & 229 & 189 & 5 & 242 & 0.24 \\
\hline Benzyl alcohol & 13 & 205 & 128 & 5 & 248 & 0.50 \\
\hline$m$-Cresol & 12 & 202 & 153 & 5 & 308 & 0.63 \\
\hline$o$-Chlorophenol & 6 & 176 & 110 & 5 & 340 & 0.63 \\
\hline Diphenyl ether & 4 & 258 & 66 & 5 & 109 & - \\
\hline
\end{tabular}

${ }^{\mathrm{a}}$ The polymerization was carried out with $2 \mathrm{~g}$ of the monomer and $2 \mathrm{ml}$ of the solvent under microwave irradiation. ${ }^{\mathrm{b}}$ Final temperature of the solvent alone after $2 \mathrm{~min}$ of microwave irradiation. ${ }^{\mathrm{c}}$ Final temperature of the reaction mixture. ${ }^{\mathrm{d}}$ Measured at a concentration of $0.5 \mathrm{~g} \mathrm{dl}^{-1}$ in $m$-cresol at $30^{\circ} \mathrm{C}$. ${ }^{\mathrm{e}} \mathrm{bp}=154^{\circ} \mathrm{C} / 7$ Torr.

Table II summarizes the solvent effect on the inherent viscosity of the polyamide formed by the polycondensation of 12-aminododecanoic acid for the microwave irradiation time of 4 or $5 \mathrm{~min}$. From this table, large temperature differences were observed clearly between the final temperature of the solvent alone and the final temperature of the polymerization mixture. This is a clear indication of the participation of the polar amino acid monomer dissolved in the solvent to generate heat during the microwave irradiation polymerization. When water, that has the highest dielectric constant, was used as the solvent, the polyamide having an inherent viscosity of $0.35 \mathrm{dlg}^{-1}$ was obtained. In general, the use of the solvents having both a high dielectric constant and a high boiling point, attaining high final polymerization temperature, led to the formation of the polyamide having inherent viscosities higher than $0.5 \mathrm{dlg}^{-1}$. Suitable solvents were found to be sulfolane and amide-type solvents such as $N$-cyclohexyl-2-pyrrolidone and DMI. Hydroxyl-containing solvents having a high dielectric con-

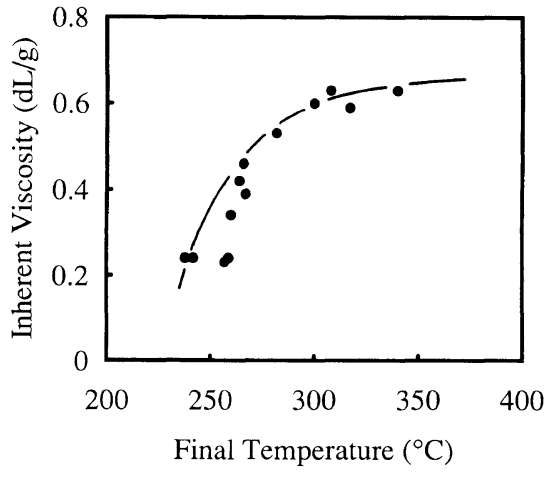

Figure 4. Relationship between final temperature and inherent viscosity of the polyamide formed by the microwave-assisted polycondensation of 12-aminododecanoic acid $(2 \mathrm{~g})$ in various solvents ( $2 \mathrm{ml})$.

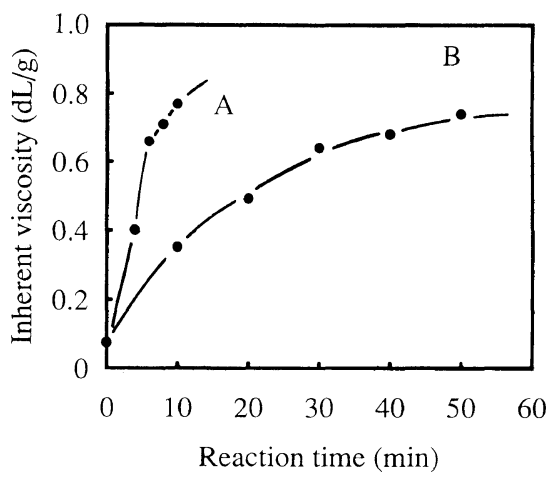

Figure 5. Time dependence of inherent viscosity of the polyamide formed by the polycondensation of 12-aminododecanoic acid $(2 \mathrm{~g})$ : (A) the microwave-assisted polycondensation in $m$-cresol, and (B) the melt polycondensation at $210^{\circ} \mathrm{C}$.

stant like ethanediol, and those with low polarity such as benzyl alcohol, $m$-cresol, and $o$-chlorophenol, were also effective for producing the polyamide with the viscosity values around $0.5 \mathrm{dlg}^{-1}$ or higher. The fact that $m$-cresol and $o$-chlorophenol were very good solvents for the aliphatic polyamides is probably related to the ready formation of the polyamide with high viscosity values. The solvent that has a high boiling point but a low dielectric constant like diphenyl ether was not heated much, probably due to low solubility of the monomer, thereby giving no polymer. Thus, the solvents played a very important role as primary efficient microwave absorbers for the microwave-assisted polycondensation.

Figure 4 exhibits the influence of the final reaction temperature on the inherent viscosity of the resultant polyamide formed by the microwave-assisted polycondensation of 12-aminododecanoic acid in various solvents, where the data are taken from Table II. It is apparent that the viscosity values were greatly affected by the final reaction temperature, and a higher final temperature afforded the polyamide having a higher viscosity value. In general, the final temperature above $280^{\circ} \mathrm{C}$ was required to obtain this type of polyamide with an inherent viscosity higher than $0.5 \mathrm{dl} \mathrm{g}^{-1}$.

Figure 5 shows a comparison of the time dependence curve of the inherent viscosity of the polyamide formed by the microwave-assisted polycondensation of 12 aminododecanoic acid with that by the conventional melt polycondensation. It is evident that the microwaveassisted polycondensation for curve A proceeded much 
Table III. Microwave-assisted synthesis of various polyamides from both $\omega$-amino acids and nylon salts ${ }^{\mathrm{a}}$

\begin{tabular}{|c|c|c|c|c|}
\hline \multirow{2}{*}{\multicolumn{2}{|c|}{ Polymer type }} & \multicolumn{3}{|c|}{ Polymer $\eta_{\mathrm{inh}}^{\mathrm{b}} / \mathrm{dl} \mathrm{g}^{-1}$} \\
\hline & & $m$-Cresol & $\mathrm{DMI}^{\mathrm{c}}$ & Sulfolane ${ }^{\mathrm{d}}$ \\
\hline \multirow[t]{11}{*}{ Nylon } & 6 & 0.50 & 0.52 & 0.41 \\
\hline & 11 & 0.58 & 0.59 & 0.54 \\
\hline & 12 & 0.35 & 0.62 & 0.48 \\
\hline & 66 & 0.27 & 0.29 & 0.17 \\
\hline & 68 & 0.22 & 0.24 & 0.26 \\
\hline & 610 & 0.20 & 0.21 & 0.21 \\
\hline & 612 & 0.24 & 0.25 & 0.21 \\
\hline & 126 & 0.58 & 0.49 & 0.57 \\
\hline & 128 & 0.33 & 0.36 & 0.32 \\
\hline & 1210 & 0.52 & 0.36 & 0.51 \\
\hline & 1212 & 0.41 & 0.39 & 0.33 \\
\hline
\end{tabular}

a The polymerization was carried out with $2 \mathrm{~g}$ of the $\omega$-amino acid or nylon salt and $1 \mathrm{ml}$ of the solvent under microwave irradiation for 4 min. ${ }^{b}$ Measured at a concentration of $0.5 \mathrm{~g} \mathrm{dl}^{-1}$ in $m$-cresol at $30^{\circ} \mathrm{C}$. 'DMI: 1,3-dimethylimidazolidone, and the polymerization was conducted for $5 \mathrm{~min}$. ' Sulfolane: tetramethylene sulfone.

Table IV. Solvent effect on microwave-assisted polycondensation of 612 nylon salt ${ }^{\mathrm{a}}$

\begin{tabular}{|c|c|c|c|c|c|c|}
\hline \multicolumn{4}{|l|}{ Solvent } & \multicolumn{2}{|c|}{ Reaction } & \multirow{2}{*}{$\begin{array}{c}\text { Polymer } \\
\eta_{\text {inh }}{ }^{\mathrm{d}}\end{array}$} \\
\hline \multirow{2}{*}{ Type } & \multirow{2}{*}{$\varepsilon$} & bp & $\mathrm{Ft}^{\mathrm{b}}$ & Time & $\mathrm{Ft}^{\mathrm{c}}$ & \\
\hline & & $\mathrm{C}$ & ${ }^{\circ} \mathrm{C}$ & $\min$ & ${ }^{\circ} \mathrm{C}$ & $\mathrm{dl} \mathrm{g}^{-1}$ \\
\hline Water & 78 & 100 & 97 & 3 & 305 & 0.21 \\
\hline Tetramethylene sulfone & 43 & 287 & 224 & 4 & 305 & 0.23 \\
\hline Dimethylacetamide & 38 & 166 & 163 & 3 & 317 & 0.15 \\
\hline 1,3-Dimethylimidazolidone & 37 & 225 & 202 & 4 & 298 & 0.27 \\
\hline$N$-Methylpyrrolidone & 32 & 202 & 179 & 4 & 313 & 0.25 \\
\hline Nitrobenzene & 35 & 211 & 198 & 3 & 286 & 0.21 \\
\hline Ethanediol & 38 & 197 & 193 & 3 & 303 & 0.20 \\
\hline 1,4-Butanediol & 31 & 229 & 189 & 5 & 279 & 0.24 \\
\hline$m$-Cresol & 12 & 202 & 153 & 4 & 310 & 0.24 \\
\hline$o$-Chlorophenol & 6 & 176 & 110 & 4 & 280 & 0.29 \\
\hline
\end{tabular}

${ }^{a}$ The polymerization was carried out with $2 \mathrm{~g}$ of the nylon salt and $2 \mathrm{ml}$ of the solvent under microwave irradiation. ${ }^{\mathrm{b}}$ Final temperature of the solvent alone after $2 \mathrm{~min}$ of microwave irradiation. ${ }^{\mathrm{c}}$ Final temperature of the reaction mixture. ${ }^{\mathrm{d}}$ Measured at a concentration of $0.5 \mathrm{~g} \mathrm{dl}^{-1}$ in $m$-cresol at $30^{\circ} \mathrm{C}$

faster than the melt polycondensation for curve B. Thus it is concluded that the internal heat generation of both the solvent and monomer under the microwave irradiation was much more effective for the progress of the polycondensation, producing the polyamide having a high viscosity value in a shorter polymerization time, than the conventional external heating.

The microwave-assisted polycondensation was extended to the polycondensation of the $\omega$-amino acids such as 6-aminohexanoic acid and 11-aminoundecanoic acid in addition to 12-aminododecanoic acid. Table III summarizes the results of the microwave-assisted synthesis of various polyamides. All three amino acids readily afforded the polyamides having reasonable inherent viscosities around $0.5 \mathrm{dlg}^{-1}$ in such solvents as $m$-cresol, DMI, and sulfolane after only $4 \mathrm{~min}$ of the microwave irradiation.

\section{Polyamide Synthesis from Nylon Salts}

Table IV summarizes the solvent effect on the inherent vicosity of the polyamide by the polycondensation of 612 nylon salt composed of hexamethylenediamine and dodecanedioic acid for the microwave irradiation time of 3-5 min. The heat generation effect of 612 nylon salt was much higher than that of 12-aminododecanoic acid (Table II), going up to the temperature above $280^{\circ} \mathrm{C}$, regardless of the kind of the solvents used. In this case, no appreciable solvent effect was observed with respect to the viscosity values of the polyamide formed, and the organic solvents as well as water produced the polyamide having inherent viscosities in the range of $0.2-0.3 \mathrm{dlg}^{-1}$.

Table III also lists the results of the microwaveassisted synthesis of a series of polyamides starting from the nylon salts. The polycondensation of the nylon salts was again accelerated by the microwave irradiation in the presence of these polar oragnic solvents, yielding the polyamides having viscosity values between 0.2 and $0.5 \mathrm{dlg}^{-1}$. In more detail, the hexamethylenediaminebased nylon salts such as 66, 68, 610, and 612 afforded the polyamides with rather lower viscosity values around $0.2 \mathrm{dl} \mathrm{g}^{-1}$, whereas the dodecamethylenediamine-bearing nylon salts such as $126,128,1210$, and 1212 gave the polyamides with higher inherent viscosities in the range of $0.3-0.6 \mathrm{dl} \mathrm{g}^{-1}$. The hexamethylenediamine-based nylon salts tended to discolor partly during the microwave heating, causing the lowering of the diamine functionality, while such a discoloration was not observed for the polycondensations of the dodecamethylenediamine-bearing nylon salts. Therefore, this may be a major reason for the low viscosity of the polyamides derived from hexamethylenediamine. In addition, hexamethylenediamine has a lower boiling point than dodecamethylenediamine, and the former may evaporate more readily during the polymerization, leading to a change in stoichiometry between the diamine and the dicarboxylic acids. This may also play a role in part for the formation of the polyamides with lower viscosity values.

\section{CONCLUSIONS}

We have developed a new facile method for the rapid synthesis of aliphatic polyamides by using a domestic microwave oven by the polycondensation of both $\omega$ amino acids and nylon salts in the presence of a small amount of a polar organic medium that acts as a primary microwave absorber. The use of such an organic medium was necessary to induce effective homogeneous heating of the monomers and thereby subsequent polycondensation leading to the formation of the polyamides having high inherent viscosities around $0.5 \mathrm{dlg}^{-1}$ or above. The microwave-assisted polycondensation proceeded rapidly, compared with the conventional melt polycondensation, and was almost complete within $5 \mathrm{~min}$. This method should become more and more important from the viewpoint of high efficieny utilization of heat energy.

\section{REFERENCES}

1. M. Teffal and A. Gourdenne, Eur. Polym. J., 19, 543 (1983).

2. D. Al Doori, R. Huggett, J. F. Bates, and S. C. Brooks, Dent. 
Mater., 4, 25 (1988).

3. J. O. Stoffer and S. P. Sitaram, Am. Chem. Soc., Proc. Polym. Mater. Sci. Eng., 71, 55 (1994).

4. J. Mijovic and J. Wijaya, Polym. Compos., 11, 184 (1990).

5. F. M. Thuillier, H. Jullien, and M. F. Grenier-Loustalot, Polym. Commun., 27, 206 (1986)

6. D. A. Lewis, J. C. Hedrick, J. E. McGrath, and T. C. Ward, Polym. Prepr., Am Chem. Soc., Div. Polym Chem., 28 [2], 330 (1987).

7. Q. Le Van and A. Gourdenne, Eur. Polym. J., 23, 777 (1987).

8. N. Beldjoudi, A. Bouazizi, D. Douibi, and A. Gourdenne, Eur. Polym. J., 24, 49 (1988).

9. S. M. Singer, J. Jow, J. D. Delong, and M. C. Hawley, SAMPE Quat., 20, 14 (1989).

10. J. Mijovic and J. Wijaya, Macromolecules, 23, 3671 (1990).

11. B. Silinski, C. Kuzmycz, and A. Gourdenne, Eur. Polym. J., 23, 273 (1987).
12. H. Jullien and H. Valot, Polymer, 26, 506 (1985).

13. D. A. Lewis, J. D. Summers, T. C. Ward, and J. E. McGrath, J. Polym. Sci., A, Polym. Chem., 30, 1647 (1992).

14. V. S. Kishanprasad and P. H. Gedam, J. Appl. Polym. Sci., 50, 419 (1993).

15. K. H. Park, S. Watanabe, M. Kakimoto, and Y. Imai, Polym. $J ., 25,209$ (1993).

16. S. Watanabe, K. Hayama, K. H. Park, M. Kakimoto, and Y. Imai, Makromol. Chem., Rapid Commun., 14, 481 (1993).

17. Y. Imai, H. Nemoto, S. Watanabe, and M. Kakimoto, Polym. Prepr., Jpn., 43, 377 (1994).

18. Y. Imai, Polym. Prepr., Am. Chem. Soc., Div. Polym. Chem., 36 [1], 711 (1995).

19. W. R. Sorenson and T. W. Campbell, "Preparative Methods of Polymer Chemistry," 2nd ed, Interscience, New York, N. Y., 1968, p 74.

20. C. D. Papaspyrides, Polymer, 29, 114 (1988). 